\title{
The Antibiotic Resistance Pattern of Gram-Negative Bacteria in Children Younger Than 24 Months with a Urinary Tract Infection: A Retrospective Single-Center Study over 15 Consecutive Years
}

\author{
Yoon Kyoung Lee, M.D. ', \\ Haejeong Lee ${ }^{2}$, \\ Jong Min Kim, M.D.', \\ Ji-Man Kang, M.D. ', \\ Sang Taek Lee, M.D. ${ }^{1}$, \\ Nam Yong Lee, M.D. ${ }^{3}$, \\ Yae-Jean Kim, M.D. ', \\ Heeyeon Cho, M.D. ${ }^{\prime}$ \\ ${ }^{1}$ Department of Pediatrics, Samsung \\ Medical Center, Sungkyunkwan University \\ School of Medicine, Seoul, Korea. \\ ${ }^{2}$ Samsung Biomedical Research Institute, \\ Sungkyunkwan University School of \\ Medicine, Seoul, Korea. ${ }^{3}$ Department \\ of Laboratory Medicine and Genetics, \\ Samsung Medical Center, Sungkyunkwan \\ University School of Medicine, Seoul, Korea \\ Corresponding author: \\ Heeyeon Cho, M.D. \\ Department of Pediatrics, Samsung Medical \\ Center, Sungkyunkwan University School of \\ Medicine, 81 Irwon-ro, Gangnam-gu, Seoul \\ 135-710, Republic of Korea \\ Tel: +82-2-3410-3539 \\ Fax: +82-2-3410-0043 \\ E-mail:choheeyeon@gmail.com
}

Received: 15 September 2015

Revised: 13 October 2015

Accepted: 25 October 2015

\begin{abstract}
This is an open-access article distributed under the terms of the Creative Commons Attribution Non-Commercial License (http:// creativecommons.org/licenses/bync/3.0/) which permits unrestricted non-commercial use, distribution, and reproduction in any medium, provided the original work is properly cited.
\end{abstract}

Purpose: We investigated trends in antibiotic resistance for gram-negative bacteria in infants with a urinary tract infection (UTI) over 15 years at a single institution.

Methods: A retrospective chart review was conducted for children younger than 24 months who visited the emergency room and were diagnosed with a UTI between January 2000 and December 2014. We selected urine culture data that grew Escherichia coli and Klebsiella pneumoniae. Baseline clinical information and results of antimicrobial susceptibility tests were analyzed by dividing the 15-year study time frame into three periods (A: 2000-2004, B: 2005-2009, and C: 20102014).

Results: During the study period, 478 applicable children were identified ( $E$. coli, 89.7\% and K. pneumoniae, 10.3\%). Antibiotic resistance to third-generation cephalosporins was increased from period $A$ to period $C(A, 2.1 \%$; $B, 8.3 \% ; C, 8.8 \%$; $P=0.025)$. Resistance to quinolones also showed a steady pattern during periods A to $C$, although it was not statistically significant $(A, 7.9 \% ; B, 9.7 \% ; C, 12.4 \%$; $P=0.221)$. The incidence of Extended-spectrum $\beta$-lactamase (ESBL)-producing gram-negative bacteria increased from period $A$ to period $C(A, 1.4 \% ; B, 7.6 \% ; C$, $8.2 \% ; P=0.012$ ).

Conclusion: This study revealed that the common uropathogens $E$. coli and $K$. pneumoniae experienced increasing resistance rates against third-generation cephalosporins and a constant antibiotic resistance to quinolones in children younger than 24 months. We also showed a recent increased incidence of ESBLproducing gram-negative bacteria in patients with community-acquired UTIs. Therefore, it is necessary to actively surveil resistance in order to properly select empirical antibiotics.

Key words: Urinary tract infection, Quinolone, Extended-spectrum $\beta$-lactamase

\section{Introduction}

Urinary tract infection (UTI) is one of the most common bacterial infections in children and infants and is a common cause of fever in infants ${ }^{1)}$. Most patients have a good prognosis after appropriate antibiotic treatment; however, most UTIs require early diagnosis and appropriate treatment, as some 
types of renal injuries in infants can lead to long-term complications such as renal insufficiency, hypertension, or chronic renal failure as an adult ${ }^{1,2)}$. Therefore, if UTI is clinically suspected in patients with fever who present to the hospital, empirical antibiotics should be started before urine culture results are finalized in order to decrease the possibility of sepsis or renal-associated complications ${ }^{1,2)}$.

Escherichia coli (E. coli), Klebsiella pneumoniae ( $K$. pneumonia), Proteus spp, Citrobacter spp, and Enterococcus spp are common causes of UTI; E.coli is the most common pathogen, accounting for $65 \sim 90 \%$ of UTI children ${ }^{1)}$. Although antibiotics such as ampicillin or amoxicillin are used as first-line treatment agents because of the high $E$. coli sensitivity to beta-lactam antibiotics, increasing resistance to these antibiotics has been reported in several studies $^{3,4)}$. In addition, cephalosporin and quinolone antibiotics seem to have emerged as the main problem of multi-drug resistance ${ }^{3,4)}$. Quinolone-resistant strains have been found in spite of age restrictions on their use in Korea ${ }^{5}$. In addition, extended spectrum $\beta$-lactamase (ESBL) producing $E$. coli, which can hydrolyze antibiotics such as penicillin, has shown increasing resistance to third-generation cephalosporins and monobactams ${ }^{6}$.

Urinary tract infection caused by antibiotic-resistant bacteria has led to problems such as inappropriate antibiotic treatment, increasing complications, and extended duration of treatment ${ }^{2,3)}$. Therefore, we investigated trends in antibiotic resistance for gram-negative bacteria in children who have been diagnosed with urinary tract infection (UTI) over a 15-year period. We also explored trends in resistance rates of third-generation cephalosporins and quinolones and the frequency of ESBL-producing E. coli based on urine culture test results in pediatric patients younger than 24 months of age.

\section{Materials and methods}

\section{Study population}

All patients who visited our institution's pediatric emergency room between January 2000 and December 2014 were selected from our medical record database. The inclusion criteria were: (a) age younger than 24 months and (b) E. coli or Klebsiella pneumoniae (K. pneumoniae) on urine culture. We excluded some patients with urinary tract anomalies such as bilateral hydronephrosis, ureteropelvic obstruction, or vesico-ureteral reflux. Urinary tract infection was defined by the following: (a) fever higher than 38 degrees on a tympanic thermometer and not due to other causes; (b) leukocyte esterase- or nitrite-positive on urinalysis or more than five white blood cells (WBC) per high-power field on urine sediment microscopy, and (c) more than $10^{5}$ colony forming units (CFU)/ml on urine culture analysis. Urine collection was attempted using a urine catheter or an aseptic urine bag if the catheter method failed.

\section{Study protocol}

Demographic characteristics, clinical and laboratory findings, causative strain, and the antibiotic resistant rate of patients with community-acquired UTI were retrospectively analyzed using the electronic medical record in order to investigate the ages of participants. Target children were then divided into two age groups (0-12 months, 13-23 months). We evaluated the resistance rates of $E$. coli and $K$. pneumoniae to ampicillin, ampicillin/sulbactam, thirdgeneration cephalosporins, piperacillin/tazobactam, amikacin, trimethoprim/sulfamethoxazole (TMP/SMX), and quinolones (ciprofloxacin, levofloxacin). We also examined the presence of ESBL-producing strains. Intermediate sensitivity on antibiotic susceptibility test was considered antibiotic resistance. Antibiotic susceptibility and ESBL-producing strains were measured using the VITEK 2 (Bio-Merieux, Durham, NC, USA).

\section{Statistical analysis}

All statistical analyses were performed with SPSS software (version 18.0 for Windows; SPSS, Chicago, IL). Results with a P value less than .05 were considered statistically significant. The Chi-square test was used for categorical variables, and a logistic regression model was used for noncategorical data.

\section{Results}

\section{Clinical characteristics}

A total of 891 patients were found to harbor E. coli or $K$. 
pneumoniae on urine culture during the 15 -year study period. We analyzed 478 patients and excluded those with underlying anomalies, for a total of 413 patients. Among 478 patients, E. coli was identified in 429 patients (89.7\%) and K. pneumoniae in 49 patients (10.3\%). There were 147 male patients (30.8\%) and 331 female patients (69.2\%). The mean age was 1.4 years ( $0.0 \sim 1.9$ years range). When divided into two age groups ( 0 - 12 months and $13-23$ months), the younger group (434 people, $90.8 \%$ ) was larger than the older group (44 people, 9.2\%). The number of patients who attended a day care center and had a history of antibiotics use were $13(2.7 \%)$ and 32 (6.7\%) in patients with communityacquired UTI, respectively. The number of patients who underwent renal ultrasonography was 337 (70.5\%) (Table 1).

\section{Strain-specific antibiotic resistance rates}

The antibiotic resistance rates to E. coli and K. pneumoniae cultured from urinalysis were examined. When analyzing the number of patients with resistance to each antibiotics, we found $72.4 \%$ resistance rate to ampicillin (346 cases), $25.1 \%$ to ampicillin/sulbactam (120 cases), 6.7\%

\begin{tabular}{|c|c|}
\hline Variable & Number (\%) \\
\hline Patients with age of $<12$ months & $434(90.8)$ \\
\hline Male: Female & 147: 331 \\
\hline \multicolumn{2}{|l|}{ Previous Antibiotic Use } \\
\hline None & $341(71.3)$ \\
\hline Within one week & $21(4.4)$ \\
\hline Within one month & $11(2.3)$ \\
\hline \multicolumn{2}{|l|}{ Urinalysis } \\
\hline Pyuria & $348(72.8)$ \\
\hline Leukocyte esterase & $346(72.4)$ \\
\hline
\end{tabular}

* Definition of fever: $\geq 38.0^{\circ} \mathrm{C}$ as measured by ear thermometer to third-generation cephalosporins (32 cases), $11.1 \%$ to piperacillin/tazobactam (53 cases), $0.8 \%$ to amikacin (4 cases), $31.6 \%$ to TMP/SMX (151 cases), and $10.3 \%$ to quinolones ( 49 cases). ESBL production was also found in 29 patients (6.1\%). In particular, when we analyzed the antibiotic resistance of the isolated $E$. coli, resistance to third-generation cephalosporins was found in $6.8 \%$ of isolates ( 29 cases), and $11.4 \%$ of isolates ( 49 cases) were resistant to quinolones (ciprofloxacin or levofloxacin). ESBL production was identified in $6.1 \%$ (26 cases) of isolated E. coli. For K. pneumoniae, there was a $6.1 \%$ resistance rate to third-generation cephalosporins ( 3 cases) and $0.0 \%$ to quinolones ( 0 cases). The ESBL production rate was $6.1 \%$ (3 cases) of isolated $K$. pneumoniae cultures (Table 2).

\section{Trend of periodic antibiotic resistance}

When divided into three time periods, period A (2000 2004), period B (2005 2009), and period C (2010 2014), resistance rates showed a changing trend to third-generation cephalosporins, with a significant increase up to 2014 $(P<0.05)$. The resistance rates to ampicillin-sulbactam significantly decreased $(P=0.000)$. However, antibiotic resistance rates to quinolones increased by $7.9 \%$ in period $\mathrm{A}$ (11/140), by $9.7 \%$ in period B (14/144), and by $12.4 \%$ in period $C$ (24/194); these changes were not statistically significant $(P>$ 0.05). The ratio of gram-negative bacteria producing ESBL significantly increased to $1.4 \%$ in period $\mathrm{A}$, to $6.7 \%$ in period $\mathrm{B}$, and to $8.2 \%$ in period $\mathrm{C}(P<0.05)$ (Fig. 1$)$.

\section{Discussion}

In this study, there was no difference in the pathogens

Table 2. The Frequency (\% of total) of Antibiotic Resistance of Pathogens

\begin{tabular}{lccc}
\hline Antibiotics & Total, N (\%) & E.coli, N (\%) & K. pneumoniae, N (\%) \\
$(\mathrm{N}=478)$ & $(\mathrm{N}=429)$ & $297(69.2)$ & $49(100.0)$ \\
\hline Ampicillin & $346(72.4)$ & $117(27.3)$ & $3(6.1)$ \\
Ampicillin/Sulbactam & $120(25.1)$ & $29(6.8)$ & $3(6.1)$ \\
Third-generation Cephalosporin & $32(6.7)$ & $46(10.7)$ & $7(14.3)$ \\
Piperacillin/Tazobactam & $53(11.1)$ & $147(34.3)$ & $4(8.2)$ \\
Trimethoprim/Sulfamethoxazole & $151(31.6)$ & $3(0.7)$ & $1(2.0)$ \\
Amikacin & $4(0.8)$ & $49(11.4)$ & $0(0.0)$ \\
Quinolone & $49(10.3)$ & $26(6.1)$ & $3(6.1)$ \\
Extended-Spectrum $\beta$-Lactamase & $29(6.1)$ & & \\
\hline
\end{tabular}




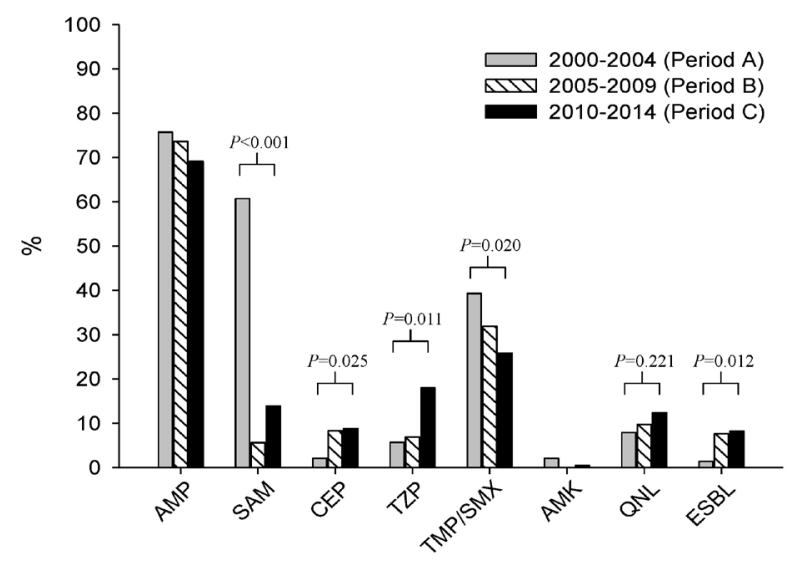

Fig. 1. Trend in Antibiotic Resistance Over 15 Consecutive Years Abbreviations: AMP, Ampicillin; SAM, Ampicillin-Sulbactam; CEP, Third-generation cephalosporins; TZP, Piperacililin-Tazobactam; TMP/SMX, Trimethoprim/Sulfamethoxazole; AMK, Amikacin; QNL, Quinolone; ESBL, Extended-Spectrum $\beta$-Lactamase

isolated on urine culture (E. coli, 89.7\% and K. pneumoniae, 10.3\%) compared with a previous study ${ }^{7}$. When examined over three periods of 2000 to 2004, 2005 to 2009 , and 2010 to 2014, the antibiotic resistance rates to third-generation cephalosporin and the ratio of ESBL-producing gramnegative bacteria significantly increased. In addition, the trend in antibiotic resistance to quinolones showed a steady distribution although it was not statistically significant. In a previous study with children older than 24 months, a trend of antibiotic resistance to third-generation cephalosporins and quinolones decreased over two periods, i.e., 2003-2005 and 2006-2008, different from our results with children younger than 24 months. We suggest that the results of this study reflect the impact of more frequent antibiotics use from 2000-2014. Recently, the increased use of thirdgeneration cephalosporin antibiotics for the treatment of urinary tract, respiratory tract, or gastrointestinal tract infections have led to increased local antibiotic resistance rates in urinary tract infections ${ }^{8)}$. According to another study, the rate of antibiotic prescriptions for respiratory infections in children decreased to $55.4 \%$ from $60.5 \%$ from 2009 to 2011; however, it has been reported that the thirdgeneration cephalosporin antibiotic prescription rate increased from $4.2 \%$ to $6.0 \%{ }^{9}$.

In adults, fluoroquinolone-based or third-generation cephalosporin antibiotics have been recommended as the initial primary drug for treatment of urinary tract infections ${ }^{10)}$. However, quinolone antibiotic-resistant strains are increasing in community-acquired infections, leading to a clinical problem. According to a recent study with adults, the ciprofloxacin resistance rate in E. coli was $28.4 \%$, and it was found to be $20 \%$ in community-acquired acute pyelonephritis cases from 2010 to 2012 [11]. In the present study involving children younger than 24 months, the overall quinolone resistance rate was 11.4\% (49/429) in $E$. coli; when analyzed according to time period, the rate was $7.9 \%$ in the A group (2000-2004), 9.7\% in the B group (2005 to 2009), and $12.4 \%$ in the $C$ group (from 2010 to 2014). Therefore, there was an approximately $10 \%$ antibiotic resistance rate in infants who did not have a history of quinolone exposure.

The use of quinolone antibiotics has been limited in pediatric patients because of the risk of joint cartilage damage shown in animal studies and the increasing risk of antibiotic resistance. However, according to accumulated research results, the United States FDA (Food and Drug Administration) and the EMA (European Medicines Agency) approved quinolones for some infectious diseases (pneumonia caused by Pseudomonas aeruginosa in patients with cystic fibrosis, chronic suppurative otitis media, complicated urinary tract infections, etc. $)^{5)}$. For local use, quinolone antibiotics are still restricted. The quinolone antibiotics prescription rate has been reported to have significantly decreased to $0.26 \%$ from $4.81 \%$ in children since the Korea Pharmaceutical Management Service contraindicated the extensive use of quinolone antibiotics in children younger than 18 years through the DUR (Drug Utilization Review) Notice in December 2009 ${ }^{12}$. Therefore, other factors must be considered to explain antibiotic resistance in infants who do not have a history of antibiotic exposure and the steady increase in resistance over time. Patients were excluded from this study if they had risks associated with increasing antibiotic resistance (previous quinolone antibiotic use, hospitalization history, frequent urinary tract infections, invasive procedure prior to catheter placement, and chronic diseases such as neurological disorders) ${ }^{11)}$. Some studies on antibiotic resistance rates in pediatric patients without antibiotic exposure have suggested that quinolone antibiotic resistance in children is associated with a gene derived from a non-pathogenic 'qnr' bonded to the E. coli pathogen or K. pneumoniae (conjugation). These researchers posited 
that the source of these genes is horizontal transmission from a different reservoir such as adults, animals, or water ${ }^{13,14)}$. This could lead to resistance through multiple propagation factors even if there was no exposure to quinolone antibiotics. Epidemiological research is also needed to demonstrate other community risk factors. In addition, efforts evaluating the proper use of quinolone antibiotics in adults are necessary as increasing antibiotic consumption in adult patients can lead to childhood antibiotic resistance.

The incidence of ESBL-producing gram-negative bacteria has increased rapidly over the past 10 years; it has been reported in $17.9 \%$ of UTIs in children with underlying diseases and in $10.8 \%$ of community-acquired $\mathrm{UTIS}^{15,16}$. In this study, ESBL-producing bacteria was found in $1.4 \%$, $7.6 \%$, and $8.2 \%$ of bacteria cultured in the periods 2000 2004, 2005-2009, and 2010-2014, respectively, showing a statistically significant increasing trend. All 28 patients had community-acquired UTIs without associated underlying disease, and $96.4 \%$ of these occurred in patients younger than 12 months. According to a previous study, the ratio of ESBL-producing Gram-negative bacteria decreased from $39.8 \%$ to $22.8 \%$ after extensive cephalosporin antibiotic use was limited in $2002{ }^{17)}$. It supported the more frequent use of extensive cephalosporins as an important factor in the increase in ESBL-producing gram -negative bacteria. In addition, decreased immunity, history of long-term hospitalization, and history of infection within the hospital such as a urinary tract infection are also risk factors ${ }^{18)}$. It has been reported that quinolone resistance has an association with ESBL-producing UTI pathogens. However, the exact mechanism is not yet well known, although the 'qnr' gene in the plasmid encoding for ESBL in K. pneumoniae has been found in a large number of 'qnr'-positive strains expressing $\mathrm{ESBL}^{11}{ }^{11}$. In other words, there has not yet been a clear epidemiological investigation, despite other suspected risk factors. In patients with UTIs caused by ESBL-producing gram-negative bacteria, the use of carbapenem and quinolones is recommended. This treatment requires constant monitoring and research because increasing use of such antibiotics could increase the incidence of antibioticresistant strains ${ }^{19)}$.

This study has several limitations. There were only 478 cases over 15 years; it was also limited in the ability to verify previous oral antibiotics as it was only a retrospec- tive study. These results are also specific to university hospital and might vary in local clinics or other community situations. In our study, the resistance rates to ampicillinsulbactam significantly decreased, but this change was not correlated with the change of the resistance rate to ampicillin or piperacillin-tazobactam. There was a possibility of the change in cut-off value for antibiotic sensitivity, and further evaluation is necessary.

In conclusion, third-generation cephalosporins, which have been suggested as alternative empiric antibiotics, show a statistically significant increasing trend in resistance rates. The antibiotic resistance rates of quinolones and the ratio of ESBL-producing gram-negative bacteria also increased. Therefore, before the empiric use of these antibiotics, we suggest proper follow-up and monitoring of antibiotic resistance rates for UTI in children.

\section{Financial support: None}

Conflicts of Interest: All authors declare no competing financial interests

\section{References}

1. Elder JS. Urinary tract infections. In: Kliegman RM, Stanton BF, St.Geme JW, Schor NF, Behrman RE, eds. Nelson textbook of pediatrics.19th ed. Philadelphia, PA: Elsevier; 2011:1829-34.

2. Rushton HG. Urinary tract infections in children. epidemiology, evaluation, and management. Pediatr Clin North Am 1997:44:1133-69.

3. Russo TA, Johnson JR. Proposal for a new inclusive designation for extraintestinal pathogenic isolates of Escherichia coli :EXPEC. J Infect Dis 2000;181:1753-4.

4. Russo TA, Johnson JR. Medical and economic impact of extraintestinal infections due to Eschericahia coli : focus on an increasingly important endemic problem. Microbes Infect 2003;5:449-56.

5. Choi SH, Kim EY, Kim YJ. Systemic use of fluoroquinolone in children. Korean J Pediatr 2013;56(5):196-201.

6. Hong JH, Yu JS, Lee GH. Risk factors for ciprofloxacin-resistant Escherichia coli strains in pediatric patients with acute urinary tract infection. Korean J Urol 2009;12:1219-24.

7. Jung HJ, Aum JA, Jung SJ, Huh JW. Different characteristic between Escherichia coli and non-Escherichia coli urinary tract infection. Korean J Pediatr 2007;50:457-61.

8. Yoon JE, Kim WK, Lee JS, Shin K-S, Ha T-S. Antibiotic susceptibility 


\section{www.chikd.org}

and imaging findings of the causative microorganisms responsible for acute urinary tract infection in children: a five-year single center study. Korean Journal of Pediatrics 2011;54:79-85.

9. Shin SM, Shin JY, Kim MH, Lee SH, Choi SH, Park BJ. Prevalence of antibiotic use for pediatric acute upper respiratory tract infections in Korea. J Korean Med Sci 2015;30:617-24.

10. Lee HJ, Park HC, Yoon HB, Han SS, Cha RH, Oh KH, et al. Causative organisms and antibiotics sensitivity in community acquired acute pyelonephritis. Korean J Nephrology 2008;27:688-95.

11. Kim ES, David C. Hooper. Clinical importance and epidemiology of quinolone resistance. Infect Chemother 2014;46(4):226-38.

12. Shin JY, Kim MH, Shin SM, Lee SH, Park BJ. Dramatic decrease in fluoroquinolones in the pediatric population in Korea. Pharmacoepidemiology and Drug safety 2014;23:1320-4.

13. Aihua Wang, Yonghong Yang, Quan Lu, Yi Wang, Yuan Chen, Li Deng,et al. Presence of qnr gene in Escherichia coli and Klebsiella pneumoniae resistant to ciprofloxacin isolated from pediatric patients in China. BMC Infectious Diseases 2008;8:68.

14. Ahmed AM, Motoi Y, Sato M, Maruyama A, Watanabe H, FukumotoY, et al. Zoo animals as reservoirs of gram-negative bacteria harboring integrons and antimicrobial resistance genes. Applied and environmental microbiology 2007;73(20):
Lee YK, et al. • Antibiotic Resistance in Urinary Tract Infection 153

6686-90.

15. Kim YK, Pai HJ, Lee HJ, Park SE, Choi EH, Kim JM, et al. Bloodstream Infections by Extended-spectrum $\beta$-lactamase producing Escherichia coli and Klebsiella pneumoniae in Children: Epidemiology and Clinical Outcome. Antimicrob Agents Chemother 2002;46:1481-91.

16. Lee JW, Shin JS, Seo JW, Lee MA, Lee SJ. Incidence and risk factors for extended-spectrum $\beta$-lactamase producing Escherichia coli in community-acquired childhood urinary tract infection. J Korean Soc Pediatr Nephrol 2004;8:214-22.

17. Lee JN, Pai HJ, Kim YK, Kim NH, Eun BW, Kang HJ, et al. Control of extended-spectrum $\beta$ lactamase-producing Escherichia coli and Klebsiella pneumoniae in a children's hospital by changing antimicrobial agent usage policy. Journal of antimicrobial Chemotherapy 2007;60:629-37.

18. Kim NH, Lee JA, Kim YK, Choi EH, Ha IS, Lee HJ, et al. Risk factors of urinary tract infections due to extended spectrum $\beta$-lactamase producing Escherichia coli in children. Korean J Pediatr 2004:47:164-9.

19. D.L. Paterson. Recommendation for treatment of severe infections caused by enterobacteriaceae producing extended-spectrum $\beta$-lactamases. Clin Microbiol Infect 2000;6:460-3. 